BULL. AUSTRAL, MATH. SOC.

\title{
SOME EXAMPLES OF COMPRESSIBLE GROUP ALGEBRAS
}

\section{AND OF NONCOMPRESSIBLE GROUP ALGEBRAS}

\section{KaORu Motose}

Dedicated to Professor Hisao Tominaga on his 60 th birthday.

A ring $R$ with centre $Z(R)$ is called compressible if $Z(e R e)=e Z(R) e$ for any idempotent $e$ of $R$. In this paper we shall give some examples of compressible group algebras and of noncompressible group algebras. These examples show that it is very difficult to judge the compressibility of a group algebra.

\section{Introduction}

Let $R$ be a ring and let $Z(R)$ be the centre of $R$. An idempotent $e$ of $R$ is called compressible in $R$ if $Z(e R e)=e Z(R) e$. A ring $R$ is also called compressible if every idempotent of $R$ is compressible. Such a ring has been studied by several authors (see [1-6 and 10]). In this paper we shall give examples of group algebras which are and which are not compressible. It seems to be very difficult to give characterizations of compressible modular group albegras.

Throughout this paper let $p$ be a fixed prime number, let $K$ be a field of characteristic $p$, let $G$ be a finite $p$-solvable group and let $K G$ be the group algebra of $G$ over $K$. For the subset $S$ of $G$,

Received 6 January 1986.

Copyright Clearance Centre, Inc. Serial-fee code: 0004-9727/86 $\$ A 2.00+0.00$. 
$\hat{S}$ denotes the sum of all elements of $S$ in $K G$. Let $F$ be a finite field of order $q^{r}$ where $q$ is a power of $p$ and let $A$ be a cyclic subgroup in the multiplicative group $F^{*}$ of $F$ such that $G F(q){ }^{\star} A=F^{*}$. We shall define permutations and permutation groups on $F$.

$$
\begin{aligned}
& w: x \rightarrow x^{q}, v_{a}: x \rightarrow a x \text { for } a \in A, \\
& u_{b}: x \rightarrow x+b \text { for } b \in F, W=\langle w\rangle, \\
& V=\left\{v_{a} \mid a \in A\right\}, \quad U=\left\{u_{b} \mid b \in F\right\}, \\
& G=\langle W, V, U\rangle=W V \text { and } H=\langle V, U\rangle=V U .
\end{aligned}
$$

2. Noncompressible group algebras

The purpose of this section is to show that the group algebra $K G$ is not compressible.

LEMMA 1. The element $e=|V|^{-1} \hat{V}$ is an idempotent of $K G$ and $w e=e w$ is contained in the centre Z(eKGe) of eKGe.

Proof. It is easy to see $e$ is an idempotent of $K G, H$ is a semidirect product of $U$ by $V$ and $G$ is a semidirect product of $H$ by $W$. It follows from $F^{*}=G F(q){ }_{A}$ that for every element $c$ of $F$, there exist elements $a \in A$ and $b \in G F(q)$ such that $c=a b$. Thus we have the equation $e u_{c} e=e v_{a} u_{b} v_{a}^{-1} e=e u_{b} e$. It follows from this equation that $w$ and $e u_{c} e$ commute for every element $u_{c}$ of $U$. This implies that $e w$ is contained in the centre $Z(e K G e)$ of eKGe $=e K W U e$.

The next result is the aim of this section.

PROPOSITION 1. If $r>1$, then the group algebra $K G$ is not compressible.

Proof. Assume false. Then we have from Lenma 1 that $w \hat{H}$ is contained in $Z(K G) \hat{H}$. Hence it suffices to prove $Z(K G) \hat{H}=K \hat{H}$. Let $C$ be a conjugate class of $G$ such that $\hat{C} \hat{H}$ is not zero. Since $C$ is contained in a residue class $w^{s} H$ for some $r>s \geqq 0$, it follows from 
the equation $0 \neq \hat{C} \hat{H}=|C| w^{s} \hat{H}$ that a centralizer $C_{G}\left(w^{s} h\right)$ contains a p-subgroup $U$ of $G$ for some $h \in H$. This implies $s=0$, completing the proof.

In the case $r=p=q=2, G$ is isomorphic to the symmetric group $S_{4}$ of order 24 . There exists also a group $T=S L(2,3)$ of order 24 such that $K T$ is not compressible if $p=2$.

EXAMPLE. Let $p=2$, let $Q=\langle x, y| x^{4}=1, x^{2}=y^{2}, y^{-1} x y$ $=x^{-1}>$ be the quaternion group of order 8 and let $g$ be the automorphism of $Q$ defined by $x^{g}=x y$ and $y^{g}=x$. The $\langle g>$-orbits of $Q$ are $D_{1}=\{1\}, D_{2}=\left\{x^{2}\right\}, D_{3}=\{x, x y, y\}$ and $D_{4}=x^{2} D_{3}$. Let $T$ be the semidirect product of $Q$ by a cyclic group $\langle g\rangle$ of order 3 with respect to this action and let $e=1+g+g^{2}$. Then $e$ is an idempotent and eKTe $=\sum_{i=1}^{4} K \hat{D}_{i} e$ is commutative since $x^{2}$ is central in $Q$ and $D_{4}=x^{2} D_{3}$. But $\hat{D}_{3} e$ is not contained in $Z(K T) e=\left(K \hat{D}_{1}+K \hat{D}_{2}+K\left(\hat{D}_{3}+\right.\right.$ $\left.\hat{D}_{4}\right)$ l)e. This group $T$ is isomorphic to $S L(2,3)$ and $T /\left\langle x^{2}\right\rangle$ is isomorphic to $A_{4}$. This shows $T$ is an example such that $K\left(T /\left\langle x^{2}\right\rangle\right)$ is compressible (see Proposition 2) and $K T$ is not compressible.

\section{Compressible group algebras}

In this section we shall give some examples of compressible modular group algebras.

Two idempotents $e$ and $f$ in a ring are said to be equivalent if ther exist elements $x$ and $y$ with $x y=e$ and $y x=f$. In this case, $e$ is compressible if and only if $f$ is.

Let us now again consider the permutation groups defined in the introduction, but this time let us assume that $A=F^{\star}$ and $K$ contains a primitive $n$th root of 1 where $n=|v|$. Then we shall show $K H$ is compressible where $H=V U$. Let $\left\{\theta_{1}, \theta_{2}, \ldots, \theta_{n}\right\}$ be the set of all distinct linear characters of $V$ and we set $e_{\varepsilon}=-\sum_{v \in V} \theta_{\varepsilon}\left(v^{-1}\right) v$. Then 
$\left\{e_{1}, e_{2}, \ldots e_{n}\right\}$ is the set of all primitive idempotents of $K V$ since $|V| \equiv-1(\bmod p)$ and $v e_{s}=\theta_{s}(v) e_{s}$ (see also $[1, p .236,(33.8)$ Theorem $\left.]\right)$. We start with the following lemma.

LEMMA 2. $e_{s} u e_{t} \neq 0$ and $e_{s} u e_{s}=(1-\hat{U}) e_{s}$ for every $u \in U-1$.

Proof. We should note that if $v u v^{\prime}=u$ for $u \in U-1$ and $v$, $v^{\prime} \epsilon V$, then $v=v^{\prime}=1$. Thus we can see that the coefficient of $u$ appearing in an element $e_{s} u e_{t}$ is equal to 1 . This completes the proof of the first assertion. Noting that $e_{s} v^{-1} u v e_{s}=e_{s} u e_{s}$ for all $v \in V$ by the equation $v e_{s}=e_{s} v=\theta_{s}(v) e_{s}$ and $V$ is transitive and fixed-pointfree on $U-1$, we can see that, for $u \in U-1$,

$$
e_{s}^{u e_{s}}=-\sum_{v \in V} e_{s} v^{-1} u v e_{s}=e_{s}(1-\hat{U}) e_{s}=(1-\hat{U}) e_{s} \text {. }
$$

PROPOSITION 2. Assume that $A=E^{*}$ and $K$ contains a primitive $|V|$ th root of 1 . Then $K H$ is compressible where $H=V U$.

Proof. Let $e$ be an idempotent of $K H$. Then $e$ is equivalent to an idempotent $f$ in $K V$ since the kernel of the natural homomorphism $K H \rightarrow K H / U$ is contained in the radical of $K H$. We may assume $f=\sum_{\boldsymbol{g}=1}^{m} e_{\boldsymbol{g}}$ for some $m \leqq n$. It is evident that $f Z(K H) f$ is contained in $Z(f K H f)$. Thus it remains only to prove $Z(f K H f)$ is contained in $f Z(K H)$. Let $y=\sum_{\substack{v \in V, u \in U,}} a_{v, u^{2 u}}$ be an element of $Z(f K H f)$. We should remark that $e_{s} y e_{t}=y e_{s} e_{t}=0$ for $s \neq t$ since $e_{s}=f e_{s} f$ is contained in $f K H f$. This together with Lemma 2 yields the following.

$$
\begin{aligned}
y=f y f & =\sum_{s} e_{s} y e_{s} \\
& =\sum_{s, v, u} a_{v, u} v e_{s} u e_{s} \\
& =\sum_{s, v} a_{v, 1} \theta_{s}(v) e_{s}+\sum_{s, v, u \neq 1} a_{v, u} v(1-\hat{U})_{s} \\
& =\sum_{s} c_{s} e_{s}-\sum_{s, v} d_{v} v \hat{U}_{s}
\end{aligned}
$$




$$
=z-\left(\sum_{v} a_{v} v \hat{U}\right)_{f}
$$

where $l \leqq s \leqq m, v$ and $u$ run over $V$ and $U$, respectively,

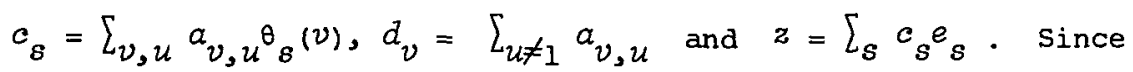
$\sum_{\nu \in V} d_{v} v \hat{U}$ is contained in $Z(K H)$, we have that $z$ is an element of $Z(f K H f)$ and so $z$ commutes with $e_{s} u e_{t}$ for all $s, t \leqq m$. It is easy to see $z=$ of for some $c \in K$ from the first assertion of Lemma 2 .

Next we shall prove that the group algebra of a finite $p$-nilpotent group over $K$ is compressible.

LEMMA 3. Let $R$ be a primary ring (see [8, p. 56]). Then $R$ is compressibze.

Proof. It follows from [8, Theorem 3.9.1] that $R$ is a full matrix ring $(S)_{n}$ of some degree $n$ over a completely primary ring $S$. Let $J(S)$ be the Jacobson radical of $S$. We considex the natural homomorphism - :R=(S) ${ }_{n} \rightarrow \bar{R}=(\bar{S})_{n}$ where $\bar{S}=S / J(S)$. The kernel of - is the radical of $R$ (see [8, Theorem 1.7.3]). Let $E_{t} \in R={ }^{(S)_{n}}$ be the matrix with 1 in the $(t, t)$ position and $0^{\prime}$ 's elsewhere. Then it follows easily that every left $\bar{R}$-module $\overline{R E}_{t}$ is irreducible and $\left\{E_{t} \mid 1 \leqq t \leqq n\right\}$ is a set of orthonormal idempotents. Let $E$ be an arbitary idempotent of $R$. Then $\overline{R E}$ is isomorphic to a direct sum of some irreducible modules $\overline{R E}_{t}$ since $\overline{R E}$ is a submodule of a completely reducible $\bar{R}$-module $\bar{R}$. And hence $E$ is equivalent to a sum of some orthogonal idempotents $E_{t}([8$, Proposition 3.7 .4 and 3.8 .1$])$. Thus we may assume $E$ is a sum of some idempotents $E_{t}$. There is a ring isomorphism between $E R E$ and $(S)_{k}$ for some $k \leqq n$ such that $Z(R) E$ is isomorphic to $Z\left((S)_{k}\right)$ under this isomorphism. This completes the proof.

Osima's theorem [9, Theorem I] together with Lemma 3 yields the following. 
PROPOSITION 3. The group algebra of a finite p-nilpotent group over $K$ is compressible.

\section{References}

[1] E.P. Armendariz, H.K. Koo and J.K. Park, "Compressible group algebras", Comm. Algebra 13 (1985), 1763-1777.

[2] E.P. Armendariz, H.K. Koo and J.K. Park, "Compressible endomorphism rings of projective modules", (preprint).

[3] E.P. Armendariz and J.K. Park, "Compressible matrix rings", BuzZ. Austral. Math. Soc. 30 (1984) 295-298.

[4] E.P. Armendariz and J.K. Park, "Compressible P.I. group algebras", (Preprint).

[5] S.K. Berberian, "The center of a corner of a ring", $J$. Algebra 71 (1981), 515-523.

[6] G.M. Bergman, "Some examples of non-compressible rings", Comm. AZgebra 12 (1984), 1-8.

[7] C.W. Curtis and I. Reiner, Representation theory of finite groups and associative algebras (Interscience, New York, 1962).

[8] N. Jacobson, The structure of pings (Amer. Math. Soc., Providence, 1956).

[9] M. Osima, "On primary decomposable group rings", Proc. Phy.-Math. Soc. Japan (3) 24 (1943), 1-9.

[10] A. Page, "Handwritten note to S.K. Berberian", (Dated 25 June 1982).

Department of Mathematics,

Faculty of Science,

Hirosaki University,

Hirosaki 036,

Japan . 